Quim. Nova, Vol. 35, No. 2, 308-314, 2012

\title{
EXTRAÇÃO SEQUENCIAL DE ZINCO E COBRE EM SOLOS TRATADOS COM LODO DE ESGOTO E COMPOSTO DE LIXO
}

\author{
Regilene Angélica da Silva Souza* \\ Departamento de Agronomia, Universidade Federal Rural de Pernambuco, Rua Dom Manuel de Medeiros, s/n, 52171-900 Recife \\ - PE, Brasil \\ Carlos Alberto Bissani, Marino José Tedesco e Rogério Chimanski da Fontoura \\ Departamento de Solos, Universidade Federal do Rio Grande do Sul, Av. Bento Gonçalves, 7712, 91540-000 Porto Alegre - RS, Brasil
}

Recebido em 27/2/11; aceito em 5/8/11; publicado na web em 30/9/11

\begin{abstract}
SEQUENTIAL EXTRACTION OF ZINC AND COPPER IN SOILS TREATED WITH SEWAGE SLUDGE AND MUNICIPAL SOLID WASTE COMPOST. This work aimed to access the contents and chemical forms and to estimate mobility and availability of cooper and zinc in samples from two soils (Haplortox and Paleudult) previously treated with doses of sewage sludge (SS) and municipal solid waste compost (MSWC), besides a control treatment. Largest percentages of $\mathrm{Cu}$ and $\mathrm{Zn}$ were determined in the organic matter fraction. $\mathrm{Zn}$ showed higher percentages of soluble and exchangeable fractions than $\mathrm{Cu}$. Treatments with SS showed higher potential of $\mathrm{Cu}$ and $\mathrm{Zn}$ availability. Modifications in soil attributes due to residue application affected metal mobility and availability indexes.
\end{abstract}

Keywords: heavy metals; organic residues; availability.

\section{INTRODUÇÃO}

A ocorrência de metais é generalizada nos solos, mas, geralmente, as concentrações desses elementos encontradas em condições naturais não representam riscos ao ambiente. Em razão de processos antrópicos, diversos estudos têm verificado o acúmulo desses elementos em solos. As atividades humanas adicionam ao ambiente materiais que contêm esses elementos, os quais podem atingir concentrações excessivas e comprometer a qualidade dos ecossistemas. As principais fontes antrópicas de metais no solo são resíduos de mineração, defensivos agrícolas, fertilizantes, lodos de estações de tratamento de esgoto urbano e/ou industrial e combustíveis fósseis. ${ }^{1,2}$

Nos últimos anos, verifica-se em todo mundo a produção crescente de resíduos urbanos, entre os quais os esgotos e o lixo domiciliar, que, quando coletados e tratados, dão origem ao lodo de esgoto e ao composto de lixo, respectivamente. Como alternativa para a disposição final desses resíduos, tem sido considerada a possibilidade de utilização na agricultura. Em vários países do mundo, inclusive no Brasil, a utilização dos resíduos orgânicos urbanos na agricultura constitui-se em prática promissora, em virtude de sua potencialidade em aumentar a fertilidade do solo, promovendo melhorias nas suas propriedades físicas, químicas e biológicas. ${ }^{3}$ A reciclagem dos resíduos em solos está fundamentada em seus conteúdos de carbono orgânico e de nutrientes.

Contudo, apesar de apresentar contribuições importantes, essa alternativa pode não representar uma prática tão segura. Esses resíduos podem conter metais, cujos efeitos potenciais podem limitar a sua utilização, devido à possível acumulação destes elementos no solo, água e plantas e subsequente entrada na cadeia alimentar. De modo geral, as concentrações nos resíduos são maiores que as naturalmente encontradas em solos. Em virtude disso, no sentido de assegurar níveis de metais que minimizem problemas potenciais para organismos do solo, plantas, seres humanos e animais, decorrentes da aplicação de resíduos urbanos, vários países estipularam, na forma de leis, limites à aplicação desses resíduos em solos agrícolas. Órgãos

\footnotetext{
*e-mail: regilenesouza@yahoo.com.br
}

governamentais, tais como a Agência de Proteção Ambiental dos Estados Unidos (USEPA) e a Comunidade Europeia, têm estabelecido normas que regulam os teores máximos de metais nos resíduos para que estes possam ser aplicados no solo. ${ }^{4,5}$ No Brasil, algumas normas e procedimentos para o uso do lodo de esgoto em áreas agrícolas têm sido estabelecidos pelo Conselho Nacional de Meio Ambiente (CONAMA), do Ministério do Meio Ambiente (MMA), bem como por outros órgãos estaduais, como a Companhia de Tecnologia de Saneamento Ambiental (CETESB), vinculada à Secretária do Meio Ambiente do Estado de São Paulo, e a Companhia de Saneamento do Paraná (SANEPAR), no Estado do Paraná. ${ }^{6-8}$

Estudos recentes têm constatado o acúmulo de metais na camada superficial do solo no decorrer de aplicações sucessivas de resíduos. ${ }^{9-11}$ Contudo, esses elementos podem estar distribuídos em diferentes formas químicas e compartimentos nos solos, conferindo-lhes diferentes graus de disponibilidade no ambiente, o que é grandemente influenciado pelas características do meio. A dinâmica dos metais pode ser influenciada por atributos do solo, tais como teores de argila e de matéria orgânica, conteúdo de óxidos e valor de $\mathrm{pH}$. A solubilidade e a disponibilidade desses elementos no sistema solo-solução-planta dependem, em grande parte, das interações com a fase sólida do solo. Estas interações são complexas, envolvendo reações de adsorção/ dessorção, precipitação/dissolução e complexação. ${ }^{12}$

A determinação das concentrações total e disponível e a estimativa da distribuição desses elementos em diversas frações dos solos, obtidas por métodos de extração sequencial de metais, têm possibilitado a avaliação de sua disponibilidade e potencial toxicidade. Embora não exista a padronização dos esquemas de extração sequencial e várias modificações venham sendo realizadas para adaptar a metodologia a condições particulares, o uso deste método permite inferências sobre a forma, o fluxo, a mobilidade e o transporte dos metais em solos..$^{13,14} \mathrm{~A}$ mesma apresenta, ainda, algumas incertezas quanto a procedimentos operacionais como, por exemplo, temperatura de secagem de amostra, tempo de contato do extrator com a amostra, método e tempo de agitação, falta de seletividade de alguns extratores e redistribuição (readsorção) dos metais entre as fases de extração. ${ }^{15}$

Desta forma, a aplicação sucessiva desses resíduos em áreas 
agrícolas tem despertado preocupação quanto aos efeitos que os metais possam exercer sobre os ecossistemas a curto e a longo prazos. Assim, tão importante quanto estudar os efeitos desses resíduos sobre as propriedades físicas e químicas dos solos é monitorar as concentrações e a distribuição destes elementos nas diferentes frações dos solos, o que possibilita estimar a sua disponibilidade e mobilidade, atuais e potenciais.

Entre os metais, geralmente, determinados nos resíduos urbanos e com concentrações expressivas tem-se o cobre e o zinco. Esses metais são considerados micronutrientes ou elementos essenciais, são requeridos pela maioria dos organismos vivos em pequenas concentrações, podendo causar toxidez quando em excesso. Assim, o presente estudo teve como objetivo avaliar, mediante extrações químicas sequenciais, a distribuição dos metais $\mathrm{Cu}$ e $\mathrm{Zn}$ nas frações: solúvel, trocável, matéria orgânica, óxidos de ferro mal cristalizados, óxidos de ferro cristalinos e residual, assim como quantificar os teores totais e disponíveis $\mathrm{Cu}$ e $\mathrm{Zn}$ e estimar sua mobilidade na camada superficial dos solos.

\section{PARTE EXPERIMENTAL}

\section{Caracterização dos solos, resíduos e tratamentos}

Foram usadas amostras de solos de um experimento previamente instalado e conduzido, com aplicação dos resíduos composto de lixo urbano (CL) e lodo de esgoto (LE).

Para a instalação do experimento, os solos foram coletados em locais sob vegetação nativa, nas camadas de 0-20 e 20-55 cm, que foram dispostas nesta mesma sequência em colunas de PVC (30 x 65 $\mathrm{cm}$ ), constituindo as unidades experimentais. Os solos utilizados foram classificados como Latossolo Vermelho Distroférrico nitossólico (LVd) e Argissolo Vermelho Distrófico arênico (PVd). ${ }^{16}$ Os resíduos orgânicos utilizados foram obtidos na Usina de Compostagem do Departamento Municipal de Limpeza Urbana (DMLU) e na Estação Piloto de Tratamento Anaeróbio de Esgoto da Vila Esmeralda, do Departamento Municipal de Água e Esgoto (DMAE), ambos da Prefeitura de Porto Alegre, RS.

Amostras da fração terra fina seca ao ar (TFSA) dos solos na profundidade de $0-20 \mathrm{~cm}$ e dos resíduos foram utilizadas para a caracterização física e química (Tabelas 1 e 2), conforme metodologias descritas em Tedesco et al.,${ }^{17}$ e para determinação dos teores de cobre e zinco. O ferro de óxidos pedogênicos (Fed) foi extraído com soluções de ditionito-citrato-bicarbonato de sódio, a $80^{\circ} \mathrm{C}$, em duas extrações sucessivas, ${ }^{18,19}$ e dos óxidos de baixa cristalinidade (Feo), com solução de oxalato de amônio $0,2 \mathrm{~mol} \mathrm{~L}^{-1}$, a pH 3,0.0

Os teores semitotais de metais nas amostras de solos e resíduos foram extraídos pelo método 3050b da Environmental Protection Agency (EPA) ${ }^{21}$ que consiste na extração da totalidade dos metais potencialmente disponíveis no ambiente. Os teores considerados disponíveis de $\mathrm{Zn}$ e $\mathrm{Cu}$ nos solos foram extraídos por solução de $\mathrm{HCl}$ 0,1 mol L ${ }^{-1} \cdot{ }^{17}$ A quantificação dos teores nos extratos foi realizada por espectrometria de absorção atômica (Perkin Elmer, modelo 2380) por atomização em chama de ar-acetileno. Nos solos, foram ainda determinados o pH em água $(1: 1)^{17}$ e o teor de carbono orgânico, por combustão seca, pelo analisador de C orgânico total (Schimadzu, modelo TCO-V csh). Em todos os procedimentos analíticos, foi incluída amostra de solo padrão de laboratório de análise de solos, como referência.

$\mathrm{O}$ experimento foi conduzido a céu aberto, em delineamento em blocos casualizados, com três repetições. À camada superficial $(0-20 \mathrm{~cm})$ de cada solo, cada material orgânico foi aplicado em três doses, em comparação com um tratamento sem adição de resíduos (testemunha). Os tratamentos estabelecidos, com as respectivas doses
Tabela 1. Caracterização da camada superficial $(0-20 \mathrm{~cm})$ dos solos LVd e PVd

\begin{tabular}{|c|c|c|}
\hline \multirow{2}{*}{ Atributo } & \multicolumn{2}{|c|}{ Valores } \\
\hline & $\mathrm{LVd}$ & PVd \\
\hline C orgânico, $\mathrm{g} \mathrm{kg}^{-1}$ & 14,0 & 5,5 \\
\hline pH em água (1:1) & 5,1 & 4,5 \\
\hline $\mathrm{P}$ disponível, $\mathrm{mg} \mathrm{dm}{ }^{-3}$ & 2,7 & 1,6 \\
\hline $\mathrm{K}$ disponível, $\mathrm{mg} \mathrm{dm}^{-3}$ & 69 & 24 \\
\hline $\mathrm{Al}$ trocável, $\mathrm{cmol}_{\mathrm{c}} \mathrm{dm}^{-3}$ & 0,4 & 0,6 \\
\hline Ca trocável, $\mathrm{cmol}_{\mathrm{c}} \mathrm{dm}^{-3}$ & 3,7 & 0,5 \\
\hline $\mathrm{Mg}$ trocável, $\mathrm{cmol}_{\mathrm{c}} \mathrm{dm}^{-3}$ & 1,6 & 0,2 \\
\hline $\mathrm{CTC}_{\mathrm{pH} 7,0}, \mathrm{cmol}_{\mathrm{c}} \mathrm{dm}^{-3}$ & 9,9 & 4,4 \\
\hline Cu semitotal, $\mathrm{mg} \mathrm{kg}^{-1}$ & 48,0 & 4,0 \\
\hline Zn semitotal, $\mathrm{mg} \mathrm{kg}^{-1}$ & 47,3 & 8,7 \\
\hline $\mathrm{Fe}_{\mathrm{d}}, \mathrm{g} \mathrm{kg}^{-1}$ & 148,8 & 9,7 \\
\hline $\mathrm{Fe}_{\mathrm{o}}, \mathrm{g} \mathrm{kg}^{-1}$ & 10,4 & 1,1 \\
\hline Argila, $\mathrm{g} \mathrm{kg}^{-1}$ & 540 & 140 \\
\hline Silte, $\mathrm{g} \mathrm{kg}^{-1}$ & 190 & 30 \\
\hline Areia, $\mathrm{g} \mathrm{kg}^{-1}$ & 270 & 830 \\
\hline
\end{tabular}

LVd: Latossolo Vermelho Distroférrico nitossólico; PVd: Argissolo Vermelho Distrófico arênico.

Tabela 2. Características físico-químicas dos resíduos

\begin{tabular}{lcc}
\hline \multirow{2}{*}{ Característica ${ }^{(1)}$} & \multicolumn{2}{c}{ Resíduo } \\
\cline { 2 - 3 } pH em água & 9,1 & 7,2 \\
Carbono orgânico, $\mathrm{g} \mathrm{kg}^{-1}$ & 83 & 210 \\
Nitrogênio total, $\mathrm{g} \mathrm{kg}^{-1}$ & 8,4 & 20 \\
$\mathrm{P}, \mathrm{g} \mathrm{kg}^{-1}$ & 3,1 & 4,4 \\
$\mathrm{~K}, \mathrm{~g} \mathrm{~kg}^{-1}$ & 8,8 & 4,4 \\
$\mathrm{Ca}, \mathrm{g} \mathrm{kg}^{-1}$ & 14 & 8,2 \\
$\mathrm{Mg}, \mathrm{g} \mathrm{kg}^{-1}$ & 5,0 & 5,5 \\
$\mathrm{~S}, \mathrm{~g} \mathrm{~kg}^{-1}$ & 1,3 & 13 \\
$\mathrm{Cu}, \mathrm{mg} \mathrm{kg}^{-1}$ & 53 & 1.195 \\
$\mathrm{Zn}, \mathrm{mg} \mathrm{kg}^{-1}$ & 152 & 766 \\
$\mathrm{Mn}, \mathrm{mg} \mathrm{kg}^{-1}$ & 373 & 224 \\
$\mathrm{Na}, \mathrm{g} \mathrm{kg}^{-1}$ & 1,2 & 0,8 \\
$\mathrm{Cr}, \mathrm{mg} \mathrm{kg}^{-1}$ & 40 & 52 \\
$\mathrm{Cd}, \mathrm{mg} \mathrm{kg}^{-1}$ & $<2$ & $<2$ \\
$\mathrm{Ni}, \mathrm{mg} \mathrm{kg}^{-1}$ & 22 & 20 \\
$\mathrm{~Pb}, \mathrm{mg} \mathrm{kg}^{-1}$ & 28 & 87 \\
$\mathrm{Hg}, \mathrm{mg} \mathrm{kg}^{-1}$ & 0,1 & 1,0 \\
\hline
\end{tabular}

${ }^{(1)}$ Determinações feitas no material seco a $75^{\circ} \mathrm{C}$, à exceção do $\mathrm{pH}$

equivalentes (base seca), foram: TES - testemunha; CL1 - $78 \mathrm{Mg} \mathrm{ha}^{-1}$; CL2 - $117 \mathrm{Mg} \mathrm{ha}^{-1}$; CL3 - $234 \mathrm{Mg} \mathrm{ha}^{-1}+$ metais (Cu e Zn); LE1 - 31 $\mathrm{Mg} \mathrm{ha}^{-1}$; LE2 - $47 \mathrm{Mg} \mathrm{ha}^{-1}$ e LE3 - $93 \mathrm{Mg} \mathrm{ha}^{-1}$ + metais (Cu e Zn). Estas doses foram estabelecidas com base no teor de $\mathrm{N}$ dos resíduos e demandas deste nutriente pelas culturas. Nos tratamentos CL3 e LE3, foram também adicionados os metais $\mathrm{Cu}$ e $\mathrm{Zn}$, na forma dos sais $\mathrm{CuSO}_{4} .5 \mathrm{H}_{2} \mathrm{O}$ e $\mathrm{ZnSO}_{4} .7 \mathrm{H}_{2} \mathrm{O}$. As quantidades dos metais adicionados na forma de sais, somadas à adição dos mesmos pelos resíduos 
orgânicos, foram calculadas para atingir as quantidades limites para aplicação em solos, sugeridas por Rodrigues et al., ${ }^{22}$ para áreas experimentais, equivalentes a $280 \mathrm{~kg} \mathrm{ha}^{-1}$ de Cu e $560 \mathrm{~kg} \mathrm{ha}^{-1}$ de $\mathrm{Zn}$.

Antes da aplicação dos resíduos, os solos foram corrigidos a pH 6,0, com base no método da solução tampão SMP, conforme a recomedação da Comissão de Química e Fertilidade do Solo - RS/SC. ${ }^{23}$ Foi utilizada uma mistura de $\mathrm{CaCO}_{3}+\mathrm{MgCO}_{3}$ em pó, na proporção molar de 3:1, com mistura manual homogênea ao solo da camada superficial $(0-20 \mathrm{~cm})$. As doses dos resíduos foram parceladas em quatro aplicações, no período de 2002-2004, antecedendo a quatro cultivos, sendo dois de verão (milho - Zea mays L.) e dois de inverno (aveia preta - Avena strigosa Schreb). Em todas as aplicações, os resíduos foram misturados à camada superficial do solo $(0-20 \mathrm{~cm})$. Maiores detalhes podem ser obtidos em $\mathrm{Kray}^{24}$ e Souza. ${ }^{25}$

As amostras dos solos foram coletadas nas unidades experimentais na camada de 0-20 cm, 2 anos após o encerramento das aplicações sucessivas dos resíduos. As amostras foram secas ao ar, peneiradas em peneiras com orifício de $2 \mathrm{~mm}$ e moídas em gral de ágata, sendo posteriormente submetidas às análises químicas.

\section{Extração sequencial}

As determinações de $\mathrm{Cu}$ e $\mathrm{Zn}$ nas frações do solo foram conduzidas com base na metodologia descrita por Silveira et al. ${ }^{26} \mathrm{e}$ Ahnstrom e Parker, ${ }^{27}$ com algumas modificações. Foram determinados os teores dos metais associados às seguintes frações: fração solúvel (FS), extraída com água destilada-deionizada; fração trocável (FT), com nitrato de estrôncio $0,1 \mathrm{~mol} \mathrm{~L}^{-1}$; fração ligada à matéria orgânica (FMO), com hipoclorito de sódio 5\%, pH 8,5; fração ligada aos óxidos de ferro mal cristalizados (FOFeMC), com uma mistura de oxalato de amônio $0,2 \mathrm{~mol} \mathrm{~L}^{-1}$ + ácido oxálico $0,2 \mathrm{~mol} \mathrm{~L}^{-1}$ a pH 3,0; fração ligada a óxidos de ferro cristalinos (FOFeC), com uma solução de oxalato de amônio $0,2 \mathrm{~mol} \mathrm{~L}^{-1}+$ ácido oxálico $0,2 \mathrm{~mol} \mathrm{~L}^{-1}(\mathrm{pH}=3,0)$ + ácido ascórbico $0,1 \mathrm{~mol} \mathrm{~L}^{-1}$; e fração residual (FR), obtida por extração pelo método $3050 \mathrm{~b}$ da Environmental Protection Agency (EPA). ${ }^{21}$ Detalhes dos procedimentos encontram-se descritos em Souza. ${ }^{25} \mathrm{~A}$ quantificação dos metais $\mathrm{Cu}$ e $\mathrm{Zn}$ nos extratos das frações extraídas foi realizada por espectrometria de absorção atômica por atomização em chama de ar-acetileno. Para cada metal, o grau de recuperação foi calculado por meio da Equação 1, sendo adotado como critério de controle os resultados obtidos na extração sequencial e estipulando-se em $\pm 15 \%$ a diferença máxima tolerável em relação ao valor semitotal.

$$
\text { Recuperação }(\%)=\frac{\Sigma[\text { Metal-frações }]}{[\text { Metal-semitotal }]} \times 100
$$

\section{Mobilidade de metais no solo}

Para estimar a mobilidade do $\mathrm{Cu}$ e $\mathrm{Zn}$ no solo, foi empregado o índice "fator mobilidade (FM)" proposto por Kabala e Singh. ${ }^{28}$ Uma vez que os metais ligados às frações FMO, FOFeMC, FOFeC e FR são menos móveis no solo, sendo mais fortemente vinculados aos constituintes do solo do que as frações FS e FT, o referido índice descreve o potencial de mobilidade dos metais no solo. Assim, este índice foi obtido a partir da Equação 2:

$$
\mathrm{FM}(\%)=\frac{(\mathrm{FS}+\mathrm{FT})}{(\mathrm{FMO}+\mathrm{FOFeMC}+\mathrm{FOFeC}+\mathrm{FR})} \times 100
$$

A análise estatística dos dados referentes aos teores semitotais e disponíveis de metais no solo foi realizada com uso do programa Sanest. ${ }^{29}$ Constou da análise de variância (teste F) e foi complementada pelo teste de comparações múltiplas de Tukey, ao nível de 5\% de probabilidade, para verificar diferenças entre as médias dos teores de metais nos diferentes tratamentos.

\section{RESULTADOS E DISCUSSÃO}

\section{Teores semitotais e disponíveis de $\mathrm{Zn}$ e $\mathrm{Cu}$}

A aplicação de doses crescentes de CL e LE e o enriquecimento dos tratamentos CL3 e LE3 com Zn e Cu contribuíram para o aumento do teor semitotal destes metais na camada de $0-20 \mathrm{~cm}$, para ambos os solos, como mostra a Tabela 3. Os maiores valores foram obtidos para os tratamentos enriquecidos com Zn e Cu (CL3 e LE3), os quais diferiram significativamente dos tratamentos testemunha, em ambos os solos. Para os demais tratamentos, apesar de em alguns casos diferirem significativamente dos seus respectivos tratamentos testemunha, as concentrações foram bem inferiores às determinadas nos tratamentos CL3 e LE3, para ambos os solos.

Tabela 3. Teores semitotais e disponíveis de $\mathrm{Zn}$ e Cu na camada superficial $(0-20 \mathrm{~cm})$, nos solos tratados com composto de lixo (CL) e lodo de esgoto

\begin{tabular}{|c|c|c|c|c|}
\hline \multirow{3}{*}{ Tratamento } & \multicolumn{2}{|c|}{$\mathrm{Zn}$} & \multicolumn{2}{|c|}{$\mathrm{Cu}$} \\
\hline & Semitotal & Disponível & Semitotal & Disponível \\
\hline & \multicolumn{4}{|c|}{ - } \\
\hline & \multicolumn{4}{|c|}{$\mathrm{PVd}$} \\
\hline TES & $7,6 \mathrm{c}$ & $1,0 \mathrm{~b}$ & $3,9 \mathrm{~d}$ & $0,9 \mathrm{~d}$ \\
\hline CL1 & $16,4 \mathrm{c}$ & $3,7 \mathrm{~b}$ & $5,4 \mathrm{~d}$ & $1,9 \mathrm{~d}$ \\
\hline CL2 & $15,5 \mathrm{c}$ & $9,5 \mathrm{~b}$ & $5,8 \mathrm{~d}$ & $2,8 \mathrm{~d}$ \\
\hline CL3 & $185,2 \mathrm{a}$ & 55,9 a & $92,8 \mathrm{a}$ & 58,4 a \\
\hline LE1 & $17,5 \mathrm{c}$ & $5,6 \mathrm{~b}$ & $18,7 \mathrm{c}$ & $10,3 \mathrm{c}$ \\
\hline LE2 & $17,4 \mathrm{c}$ & $5,5 \mathrm{~b}$ & $23,1 \mathrm{c}$ & $14,6 \mathrm{c}$ \\
\hline \multirow[t]{2}{*}{ LE3 } & $145,5 \mathrm{~b}$ & $53,3 \mathrm{a}$ & $72,3 \mathrm{~b}$ & $46,4 \mathrm{~b}$ \\
\hline & \multicolumn{4}{|c|}{$\mathrm{LVd}$} \\
\hline TES & $46,1 \mathrm{e}$ & $6,6 \mathrm{~b}$ & $47,7 \mathrm{f}$ & 8,9 e \\
\hline CL1 & $54,4 \mathrm{de}$ & $10,3 \mathrm{~b}$ & $53,9 \mathrm{e}$ & 8,6 e \\
\hline CL2 & $65,6 \mathrm{c}$ & $13,3 \mathrm{~b}$ & $57,3 \mathrm{e}$ & $9,0 \mathrm{e}$ \\
\hline CL3 & $283,2 \mathrm{a}$ & $35,9 \mathrm{a}$ & $158,9 \mathrm{~b}$ & $57,3 \mathrm{~b}$ \\
\hline LE1 & $60,3 \mathrm{~cd}$ & $10,1 \mathrm{~b}$ & $71,4 \mathrm{~d}$ & $20,7 \mathrm{~d}$ \\
\hline LE2 & $67,6 \mathrm{c}$ & $16,6 \mathrm{~b}$ & $81,9 \mathrm{c}$ & $27,5 \mathrm{c}$ \\
\hline LE3 & $254,8 \mathrm{~b}$ & $39,6 \mathrm{a}$ & $184,5 \mathrm{a}$ & $99,5 \mathrm{a}$ \\
\hline
\end{tabular}
(LE) (médias de três repetições)

Em cada solo, médias seguidas por letras iguais na coluna não diferem significativamente entre si pelo teste de Tukey $(\mathrm{p}<0,05)$.

A avaliação dos teores disponíveis de $\mathrm{Cu}$ e $\mathrm{Zn}$, utilizando o extrator $\mathrm{HCl}$ 0,1 mol L-1 (Tabela 3), mostrou que a disponibilidade dos elementos aumentou com a dose dos resíduos adicionados ao solo e com o enriquecimento do CL e $\mathrm{LE}$ com $\mathrm{CuSO}_{4} \cdot 5 \mathrm{H}_{2} \mathrm{O}$ e $\mathrm{ZnSO}_{4} .7 \mathrm{H}_{2} \mathrm{O}$, na maior dose, para ambos os solos. Com isso, verificou-se que houve diferença significativa para os tratamentos CL3 e LE3 em relação ao tratamento testemunha, para ambos os solos. Para os demais tratamentos, apesar do aumento na disponibilidade de $\mathrm{Zn}$ e $\mathrm{Cu}$, não houve diferenças significativas em relação aos seus respectivos tratamentos testemunha, para ambos os solos, com exceção do $\mathrm{Cu}$, para os tratamentos com LE.

O aporte de metais ao solo via resíduo depende tanto da concentração do metal no resíduo como da dose e da frequência de aplicação. Para o presente estudo, as quantidades totais dos metais adicionados 
em ambos os solos via resíduo e sais solúveis após as quatro etapas do experimento foram (equivalentes em $\mathrm{kg} \mathrm{ha}^{-1}$ ): $\mathrm{Zn}$ - CL1: 14,6; CL2: 22,0; CL3: 560,0; LE1: 28,9; LE2: 43,3 e LE3: 560,0; e para o $\mathrm{Cu}$ - CL1: 5,2; CL2: 7,8; CL3: 280,0; LE1: 44,6; LE2: 66,8 e LE3: 280,0. Desta forma, observa-se que os maiores aportes desses elementos foram para os tratamentos com LE. Na Tabela 2, nota-se que as concentrações de $\mathrm{Cu}$ determinadas nos resíduos foram de 1.195 e $53 \mathrm{mg} \mathrm{kg}^{-1}$ para o LE e CL, respectivamente, e de Zn, de 766 e $152 \mathrm{mg} \mathrm{kg}^{-1}$ para o LE e CL, respectivamente.

Os valores determinados nos solos não ultrapassaram os limites máximos estabelecidos pela $\operatorname{USEPA}^{5}\left(\mathrm{Zn}, 1.400 \mathrm{mg} \mathrm{kg}^{-1}\right.$ e $\mathrm{Cu}$, $\left.750 \mathrm{mg} \mathrm{kg}^{-1}\right)$ e pela norma da $\operatorname{CETESB}^{7}\left(\mathrm{Zn}, 480 \mathrm{mg} \mathrm{kg}^{-1}\right.$ e Cu , $200 \mathrm{mg} \mathrm{kg}^{-1}$ ), referentes a valores orientadores para estes elementos em solos agrícolas. Para os tratamentos sem enriquecimento, pode-se verificar que os teores encontrados são baixos, embora haja tendência de aumento com adição dos resíduos aplicados, em relação à testemunha.

\section{Distribuição de $\mathrm{Zn}$ e $\mathrm{Cu}$ nas frações do solo e fator mobilidade}

A concordância entre os valores obtidos utilizando o método 3050b e o somatório dos teores encontrados nas frações é visualizada na Tabela 4. Para o $\mathrm{Zn}$, os resultados mostram uma variação no intervalo de $102-109 \%$, com média de $105 \%$, para o PVd, e no intervalo de $93-110 \%$, com média de $103 \%$, para o LVd. Para o $\mathrm{Cu}$, os intervalos de recuperação foram de $93-109 \%$, com média de $101 \%$, para o PVd, e de $92-110 \%$, com média de $103 \%$, para o LVd. Pelo exposto, os resultados encontram-se dentro dos limites de erro tolerável estipulado $( \pm 15 \%$.). Assim, observa-se, para cada elemento, que a soma das seis frações se apresenta em concordância com a respectiva concentração semitotal determinada, em ambos os solos.

Os resultados mostram a associação do $\mathrm{Zn}$ com todas as frações do solo, para todos os tratamentos, em ambos os solos (Figura 1). Os maiores percentuais de $\mathrm{Zn}$ foram obtidos na fração matéria orgânica para o PVd, com exceção dos tratamentos com LE, em que se verifi- caram as maiores concentrações para as frações óxidos de ferro mal cristalizados (LE1 e LE2) e solúvel (LE3). Para o LVd, os maiores percentuais foram obtidos para a fração residual, com exceção dos tratamentos CL3 (fração matéria orgânica) e LE3 (fração trocável).

No geral, observa-se que nos tratamentos com CL houve aumento nos percentuais de $\mathrm{Zn}$ na fração matéria orgânica. Foram determinados percentuais de 63 e $52 \%$ de $\mathrm{Zn}$ nesta fração no tratamento CL3, para o PVd e LVd, respectivamente. Para os tratamentos que receberam LE, verificam-se acréscimos nas frações mais imediatamente disponíveis. Para o tratamento LE3, os percentuais para a fração solúvel foram de 27 e 11\%, respectivamente, para o PVd e LVd, enquanto para a fração trocável se obtiveram percentuais de 23 e $25 \%$ para o PVd e LVd, respectivamente.

No solo, o $\mathrm{Zn}$ encontra-se adsorvido a minerais e compostos orgânicos e, sobretudo, na forma de precipitados. O Zn encontra-se na forma trocável nos óxidos e argilas, e na matéria orgânica ocorre tanto complexado aos ácidos fúlvicos, quanto na forma trocável..$^{13,31}$ Em valores baixos de $\mathrm{pH}$, é adsorvido fracamente aos componentes do solo, o que lhe confere maior mobilidade. ${ }^{31}$

No presente estudo, foram observadas modificações no $\mathrm{pH}$ do solo após as aplicações sucessivas dos resíduos, em função das doses aplicadas. Nos tratamentos que receberam CL, verificou-se a manutenção dos valores de $\mathrm{pH}$ do solo, situados entre 6,0 e 6,2 no LVd e 5,7 e 5,8 no PVd (Tabela 4). Por outro lado, os tratamentos que receberam LE apresentaram um efeito acidificante, em ambos os solos, aumentando com a quantidade de lodo adicionado, atingindo valores de 3,8 e 4,7, respectivamente, para o PVd e LVd, ambos na maior dose aplicada. Este fato pode ser explicado com base nas características dos resíduos, bem como nas reações que ocorrem durante o processo de mineralização dos mesmos, como a nitrificação do nitrogênio amoniacal, ${ }^{32-34}$ a oxidação de sulfetos ${ }^{32,33}$ e a liberação de certos compostos orgânicos. ${ }^{35,36}$ Ainda cabe ressaltar as reações de hidrólise na solução do solo pela adição dos metais nos tratamentos CL3 e LE3 na forma de sais solúveis, possivelmente contribuindo para o maior abaixamento do $\mathrm{pH}$ do solo nestes tratamentos. Os

Tabela 4. Alguns atributos químicos, somatório das frações da extração sequencial, taxa de recuperação (R) e fator mobilidade (FM) dos metais Zn e Cu, determinados na camada superficial $(0-20 \mathrm{~cm})$ nos solos tratados com composto de lixo $(\mathrm{CL})$ e lodo de esgoto (LE) (médias de três repetições)

\begin{tabular}{|c|c|c|c|c|c|c|c|c|}
\hline \multirow{3}{*}{ Tratamentos } & \multirow{3}{*}{$\mathrm{pH}$} & \multirow{3}{*}{$\begin{array}{l}\text { C-org } \\
\mathrm{g} \mathrm{kg}^{-1}\end{array}$} & \multicolumn{2}{|c|}{$\sum$ Frações } & \multicolumn{2}{|c|}{$\mathrm{R}$} & \multicolumn{2}{|c|}{ FM } \\
\hline & & & $\mathrm{Zn}$ & $\mathrm{Cu}$ & $\mathrm{Zn}$ & $\mathrm{Cu}$ & $\mathrm{Zn}$ & $\mathrm{Cu}$ \\
\hline & & & \multicolumn{2}{|c|}{-------- $\mathrm{mg} \mathrm{kg}^{-1}$} & \multicolumn{4}{|c|}{ 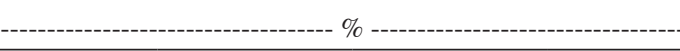 } \\
\hline & \multicolumn{8}{|c|}{ PVd } \\
\hline TES & 4,1 & 4,4 & 8,2 & 3,6 & 108 & 92 & 21,4 & 12,8 \\
\hline CL1 & 5,7 & 6,1 & 17,6 & 5,3 & 107 & 97 & 14,0 & 15,2 \\
\hline CL2 & 5,8 & 6,3 & 16,7 & 5,4 & 108 & 93 & 12,5 & 14,8 \\
\hline CL3 & 5,8 & 10,1 & 189,5 & 101,2 & 102 & 109 & 11,0 & 6,8 \\
\hline LE1 & 4,3 & 5,2 & 17,8 & 18,9 & 102 & 101 & 19,4 & 19,0 \\
\hline LE2 & 4,2 & 5,3 & 18,1 & 25,3 & 104 & 109 & 21,7 & 19,7 \\
\hline \multirow[t]{2}{*}{ LE3 } & 3,8 & 6,8 & 148,7 & 75,5 & 102 & 104 & 50,2 & 29,4 \\
\hline & \multicolumn{8}{|c|}{ LVd } \\
\hline TES & 5,0 & 15,7 & 49,0 & 50,4 & 106 & 105 & 8,3 & 2,1 \\
\hline CL1 & 6,0 & 19,6 & 60,0 & 59,1 & 110 & 110 & 5,3 & 2,5 \\
\hline CL2 & 6,2 & 20,9 & 67,0 & 60,8 & 102 & 106 & 4,1 & 2,3 \\
\hline CL3 & 6,2 & 27,3 & 271,1 & 145,9 & 95 & 92 & 6,2 & 2,1 \\
\hline LE1 & 5,5 & 16,1 & 62,5 & 75,6 & 104 & 106 & 6,5 & 2,1 \\
\hline LE2 & 5,3 & 19,1 & 73,9 & 82,4 & 109 & 101 & 7,9 & 1,8 \\
\hline LE3 & 4,7 & 22,1 & 235,9 & 171,4 & 93 & 92 & 36,9 & 4,5 \\
\hline
\end{tabular}


(A)

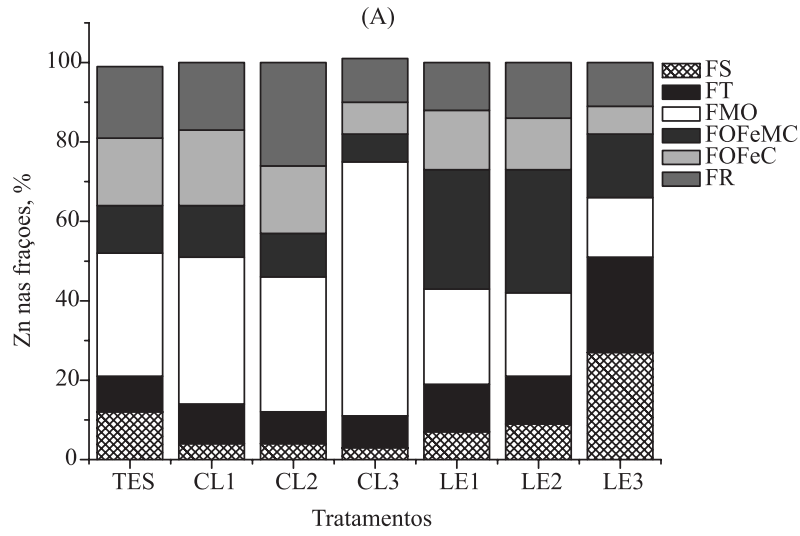

(A)

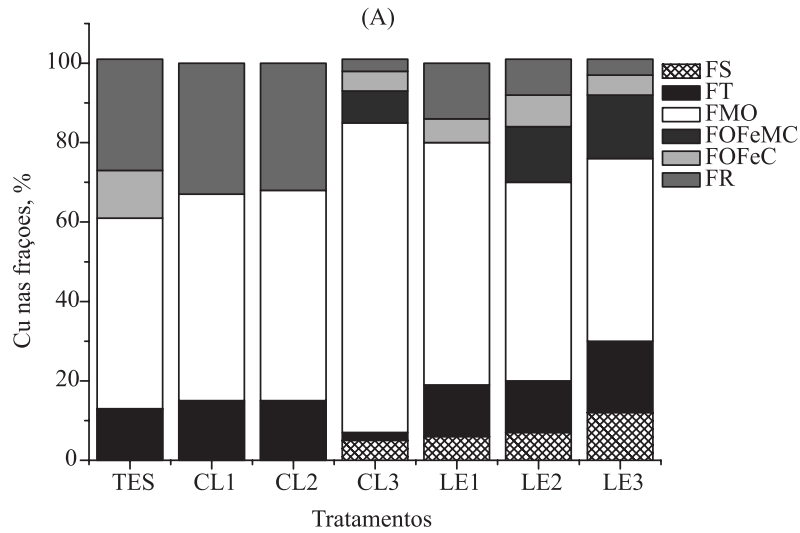

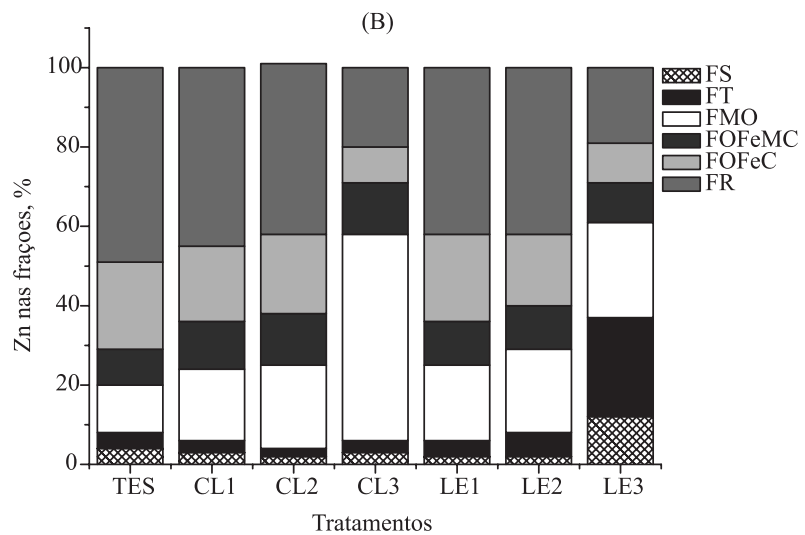

(B)

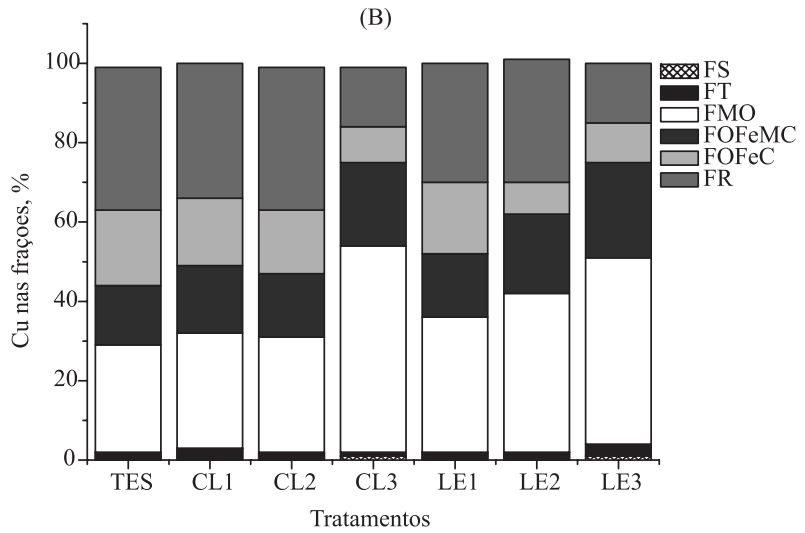

Figura 1. Distribuição relativa de Zn e Cu nas frações solúvel (FS), trocável (FT), ligado à matéria orgânica (FMO), ligado a óxidos ferro mal cristalizados (FOFeMC), ligado a óxidos de ferro cristalizados (FOFeC) e residual (FR), para os solos PVd (A) e LVd (B), em função dos tratamentos com adição de doses crescentes de composto de lixo $(C L)$ e lodo de esgoto (LE)

metais presentes na solução do solo, por meio dessas reações, liberam íons $\mathrm{H}^{+}$, afetando o $\mathrm{pH}$ do solo. Pode-se ainda considerar que a redução do $\mathrm{pH}$ do solo nos tratamentos com LE tenha contribuído para o aumento de $\mathrm{Zn}$ nas formas mais imediatamente disponíveis (FS e FT). Neste caso, o solo PVd foi o mais afetado, devido as suas características físico-químicas (Tabela 1), apresentando um menor poder tampão em comparação ao solo LVd e, portanto, as variações no $\mathrm{pH}$ são mais facilmente observadas.

Esses resultados corroboram com os de Gaivizzo, ${ }^{36}$ em experimento com aplicação de lodo industrial, em que a autora constatou aumento na participação do Zn nas formas solúvel, trocável e matéria orgânica com o aumento da quantidade de lodo aplicado e o enriquecimento dos tratamentos com Zn. Araújo et al. ${ }^{37}$ estudaram a distribuição de Zn em solos incubados com LE e observaram que os maiores teores de $\mathrm{Zn}$ foram encontrados nas frações: residual>trocável>óxidos de ferro.

Para a distribuição de $\mathrm{Cu}$, os maiores percentuais foram determinados para as frações matéria orgânica e residual, para ambos os solos estudados (Figura 1). Para o PVd, os maiores percentuais de $\mathrm{Cu}$ na fração matéria orgânica foram verificados para os tratamentos com aplicação do CL, aumentando com o aumento da dose, determinandose $78 \%$ do Cu nesta fração no tratamento CL3. Para os tratamentos com LE, verificou-se a redução na participação de $\mathrm{Cu}$ na fração matéria orgânica, com aumento da dose e, consequentemente, uma tendência no aumento dos percentuais de $\mathrm{Cu}$ nas frações solúvel e trocável. Para o LVd, foram encontrados percentuais de $\mathrm{Cu}$ de 52 e 47\% para a fração matéria orgânica, para os tratamentos CL3 e LE3, respectivamente. Para as frações consideradas mais imediatamente disponíveis, os percentuais de $\mathrm{Cu}$ aumentaram com as doses aplicadas dos resíduos, sendo os maiores valores determinados para os tratamentos com LE e para o solo PVd. Foram determinados percentuais de 12 e $18 \%$ para as frações solúvel e trocável, respectivamente, para o tratamento com LE no PVd. Nas demais frações, os percentuais de $\mathrm{Cu}$ foram baixos. Gaivizzo, ${ }^{36} \mathrm{em}$ um experimento de longa duração com adição de lodo industrial, observou aumento na participação do $\mathrm{Cu}$ nas formas orgânica e residual. Resultados semelhantes foram obtidos por Costa, ${ }^{38}$ em solos do Estado do Rio Grande do Sul, observando predomínio de maiores percentuais de $\mathrm{Cu}$ nas frações FMO e FR. Borges e Coutinho, ${ }^{39}$ em solos com aplicação de biossólido, observaram maiores percentuais de $\mathrm{Cu}$ associado à $\mathrm{FMO}$ para um solo arenoso e às frações de óxidos para um solo argiloso.

A maior retenção de $\mathrm{Cu}$ pela fração orgânica confirma a capacidade deste metal em formar complexos estáveis com a matéria orgânica, pelas reações com os grupos $\mathrm{COOH}$ e $\mathrm{OH}$ fenólicos. ${ }^{40} \mathrm{~A}$ complexação do Cu pela matéria orgânica do solo é a mais forte entre os metais de transição divalentes. Os grupos funcionais carboxílicos e fenólicos, presentes em compostos húmicos, formam estruturas carregadas negativamente, que apresentam grande afinidade pelo $\mathrm{Cu} .{ }^{41} \mathrm{~A}$ capacidade de retenção $\mathrm{Cu}$ pela matéria orgânica se deve à formação de complexos de esfera interna, também conhecida como adsorção específica. ${ }^{42}$

Observou-se que a adição dos resíduos LE e CL proporcionou aumentos significativos no teor de C orgânico nos solos, sendo este crescente com a dose (Tabela 4). Os maiores aumentos no teor de C orgânico nos solos foram obtidos para os tratamentos que receberam CL. Entre os solos, a diferença observada para os valores encontrados é devida aos teores naturais de C orgânico, que no solo LVd são aproximadamente três vezes maiores que no solo PVd (Tabela 1). 
Kray ${ }^{24}$ estudou a decomposição desses resíduos no solo, através da quantificação de $\mathrm{CO}_{2}$ liberado e taxa de degradação, verificando uma menor decomposição do CL, possivelmente devido ao processo de compostagem, resultando na maior estabilidade do material, enquanto o LE apresentou maior degradação, em virtude de ser um material ainda não completamente estabilizado. Com base nestes resultados, pode-se explicar a maior associação do $\mathrm{Cu}$ à fração orgânica para os tratamentos com CL e, possivelmente, a maior estabilidade do metal a esta fração. Em contraste, para os tratamentos com LE, em virtude de ser um material pouco estabilizado e que contribui para a diminuição do $\mathrm{pH}$ do solo para estes tratamentos, como já apresentado anteriormente, observa-se a tendência de elevação dos percentuais deste metal para as frações mais imediatamente disponíveis, o que é mais evidente para o solo PVd.

Quanto à estimativa da mobilidade desses elementos nos solos, o fator mobilidade tendeu a diminuir com o aumento das doses aplicadas para os tratamentos que receberam CL, ocorrendo o inverso para os tratamentos que receberam LE. Os maiores valores para este fator foram obtidos para o PVd. O solo PVd apresenta textura arenosa, baixas concentrações de óxidos e de C orgânico (Tabela 1) e, consequentemente, menor capacidade de reter metais. Já o solo $\mathrm{LVd}$, argiloso e com maiores teores destes constituintes, apresentou valores bem menores para este fator. Em adição, as modificações ocorridas nos atributos químicos do solo, como o observado para o $\mathrm{pH}$ do solo nos tratamentos com adição de LE, favorecem o aumento na mobilidade dos metais no solo. Conforme Ma e Rao,${ }^{43}$ os altos valores para o fator mobilidade têm sido interpretados como indicativos de alta disponibilidade de metais em solos.

Considerando o tratamento testemunha e as médias dos tratamentos que receberam LE e CL, estimou-se a sequência de mobilidade para os dois metais estudados, com base no fator mobilidade, obtendo-se, tanto para o PVd como $\mathrm{LVd}, \mathrm{Zn}>\mathrm{Cu}$. A mobilidade dos metais no solo depende das condições do meio, bem como das propriedades químicas de cada metal, tais como eletronegatividade, potencial iônico e constante de hidrólise. A adsorção competitiva de metais tem sido relatada por muitos pesquisadores, há algumas décadas. Basta e Tabatabai ${ }^{44}$ e Echeverría ${ }^{45}$ utilizaram isotermas de adsorção para explicar o comportamento competitivo entre alguns metais sob diferentes condições e tipos de solos, e verificaram maior adsorção para o $\mathrm{Pb}$ e $\mathrm{Cu}$, se comparados com $\mathrm{Cd}$, Ni e $\mathrm{Zn}$. Desta forma, o $\mathrm{Zn}$ seria considerado de maior mobilidade comparativamente ao $\mathrm{Cu}$.

No presente estudo, a extração sequencial se mostrou eficiente em inferir sobre o potencial de mobilidade e de disponibilidade de $\mathrm{Zn}$ e $\mathrm{Cu}$ nos solos tratados com CL e LE. As aplicações sucessivas destes resíduos em solos e as modificações nos atributos químicos do solo podem levar ao aumento na mobilidade destes elementos. Contudo, faz-se necessário o monitoramento constante das áreas que vêm recebendo estes resíduos e a manutenção de certos atributos do solo, como o pH, em condições favoráveis à retenção e imobilização destes elementos, com intuito de garantir a sustentabilidade de tal prática, pois, caso contrário, poderá apresentar riscos ambientais.

\section{CONCLUSÕES}

A extração sequencial permitiu que se inferisse sobre o potencial de mobilidade e de disponibilidade de $\mathrm{Zn}$ e $\mathrm{Cu}$ nos solos tratados com CL e LE, mostrando que os tratamentos que receberam LE apresentam um potencial maior de disponibilidade de $\mathrm{Zn}$ e $\mathrm{Cu}$ a curto prazo, principalmente para o solo $\mathrm{PVd}$, devido às características físico-químicas deste solo.

Os maiores percentuais de $\mathrm{Zn}$ e $\mathrm{Cu}$ nos solos ocorrem na fração orgânica. $\mathrm{O}$ metal $\mathrm{Zn}$ apresenta características de maior mobilidade no solo, podendo representar maior risco ambiental, principalmente no argissolo (PVd), de textura arenosa.

As modificações nos atributos químicos dos solos em função da aplicação dos resíduos, principalmente para os tratamentos que receberam LE, possivelmente afetaram a disponibilidade dos metais, em especial o Zn.

\section{AGRADECIMENTOS}

Ao Conselho Nacional de Desenvolvimento Científico e Tecnológico $(\mathrm{CNPq})$, pela concessão da bolsa de doutorado à primeira autora e pelo apoio financeiro ao projeto. Ao Departamento Municipal de Águas e Esgoto (DMAE) e à Usina de Compostagem do Departamento Municipal de Limpeza Urbana (DMLU), ambos da Prefeitura Municipal de Porto Alegre, por terem cedido os resíduos.

\section{REFERÊNCIAS}

1. Camargo, O. A.; Alleoni, L. R. F.; Casagrande, J. C. Em Reações dos micronutrientes e elementos tóxicos; Ferreira, M. E.; Cruz, M. C. P.; van Raij, B.; Abreu, C. A., eds.; CNPq/FAPESP/POTAFOS: Jaboticabal, 2001.

2. Costa, C. N.; Meurer, E. J.; Bissani, C. A.; Selbach, P. A. Em Contaminantes e poluentes do solo e do ambiente; Meurer, E. J., ed.; Evangraf: Porto Alegre, 2006, cap. 8.

3. Abreu Junior, C. H.; Boaretto, A. E.; Muraoka, T; Kihel, J. C. Em Uso agrícola de resíduos orgânicos potencialmente poluentes: propriedades químicas do solo e produção vegetal; Vidal-Torrado, P.; Alleoni, L. R. F.; Cooper, M.; Silva, A. P.; Cardoso, E. J., eds.; Sociedade Brasileira de Ciência do Solo: Viçosa, 2005.

4. Council of the European Communities - CEC; Council Directive 86/278/ EEC of 12 June 1986 on the protection of the environment, and in particular of the soil, when sewage sludge is used in agriculture, 1986.

5. United States Environmental Protection Agency - USEPA; Standards for the use and disposal of sewage sludge, EUA, 1993. (Code of Federal Regulations 40 CFR Part 503).

6. Companhia de Saneamento do Paraná - SANEPAR; Manual técnico para utilização agrícola do lodo de esgoto no Paraná, SANEPAR: Curitiba, 1997.

7. Companhia de Tecnologia de Saneamento Ambiental - CETESB; Aplicação de biossólido de sistemas de tratamento biológico em áreas agrícolas - Critérios para projeto e operação, CETESB: São Paulo, 1999.

8. Brasil, CONAMA; Resolução n. 375, CONAMA: Brasília, 2006.

9. Oliveira, F. C.; Mattiazzo, M. E.; Scientia Agrícola 2001, 58, 581.

10. Shober, A. L.; Stehouwer, R. C.; Macneal, K. E.; J. Environ. Qual. 2003, 32,873 .

11. Marques, M. O.; Nogueira, T. A. R.; Fonseca, I. M.; Marques, T. A.; Revista de Biologia e Ciências da Terra 2007, 7, 133.

12. Alloway, B. J.; Heavy metals in soils, Wiley: New York, 1990.

13. Sposito, G.; Lund, L. J.; Chang, A. C.; Soil Sci. Soc. Am. J. 1982, 46, 260.

14. Miller, W. P; Martens, D. C.; Zelanzny, L. W.; Soil Sci. Soc. Am. J. 1986, $50,598$.

15. Tessier, A.; Campbell, P. G. C.; Bisson, M.; Environ. Technol. 1979, 15, 844.

16. Empresa Brasileira de Pesquisa Agropecuária - EMBRAPA; Sistema Brasileiro de Classificação de Solos, CNPS: Rio de Janeiro, 1999.

17. Tedesco, M. J.; Gianello, C.; Bissani, C. A.; Bohnen, H.; Volkweiss, S. J.; Análise de solos, plantas e outros materiais, $2^{\text {a }}$ ed., UFRGS: Porto Alegre, 1995.

18. Mehra, O. P.; Jackson, M. C.; Resumos da $7^{a}$ National Conference on Clay Minerals, New York, Estados Unidos, 1960. 
19. Inda Junior, A. V.; Kämpf, N.; R. Bras. Ci. Solo 2003, 27, 1139.

20. Schwertmann, U.; Zeitung Pflanzenernähr dung Bodenkontrolle 1964, 105, 194.

21. United States Environmental Protection Agency - USEPA; Test methods for evaluating solid wastes, USEPA: Washington, 1986.

22. Rodrigues, A. L. M.; Anghinoni, M. C. M.; Tedesco, M. J.; Gianello, C.; Resumos do $22^{\circ}$ Congresso da União Internacional dos Químicos e Técnicos da Indústria do Couro, Porto Alegre, Brasil, 1993.

23. Comissão de Química e Fertilidade do Solo; Manual de adubação e calagem para os Estados do Rio Grande do Sul e de Santa Catarina, $10^{\mathrm{a}}$ ed., SBCS: Porto Alegre, 2004.

24. Kray, C. H.; Tese de Doutorado, Universidade Federal do Rio Grande do Sul, Brasil, 2005.

25. Souza, R. A. S.; Tese de Doutorado, Universidade Federal do Rio Grande do Sul, Brasil, 2009.

26. Silveira, M. L.; Alleoni, L. R. F.; O'Connor, G. A.; Chang, A. C.; Chemosphere 2006, 64, 1929.

27. Ahnstrom, Z. S.; Parker, D. R.; Soil Sci. Soc. Am. J. 1999, 63, 1650.

28. Kabala, C.; Sing, B. R.; J. Environ. Qual. 2001, 30, 485.

29. Zonta, E. P.; Machado, A. A.; SANEST - Sistema de análise estatística para microcomputadores, DMEC/IFM/UFPel: Pelotas, 1984.

30. Companhia de Tecnologia de Saneamento Ambiental - CETESB; Relatório de estabelecimento de valores orientadores para solos e águas subterrâneas no Estado de São Paulo, CETESB: São Paulo, 2005.
31. Hayes, K. F.; Traina, S. J. Em Metal speciation and its significance in ecosystem health; Huang, P. M., ed.; Soil Science Society: Madison, 1998.

32. King, L. D.; Morris, H. D.; J. Environ. Qual. 1972, 1, 425.

33. Pietz, R. I.; Carlson, C. R.; Peterson, J. R.; Zenz, D. T.; Lue-Hing, C.; J. Environ. Qual. 1989, 18, 174.

34. Boeira, R. C.; Resumos da $5^{a}$ Reunião Sul-brasileira de Ciência do Solo, Florianópolis, Brasil, 2004.

35. Chang, A. T.; Sommerfeldt, T. G.; Entz, T.; J. Environ. Qual. 1991, 20, 475.

36. Gaivizzo, L. H. B.; Tese de Doutorado, Universidade Federal do Rio Grande do Sul, Brasil, 2001

37. Araújo, J. C. T.; Nascimento, C. W. A.; R. Bras. Ci. Solo 2005, 29, 977.

38. Costa, C. N.; Tese de Doutorado, Universidade Federal do Rio Grande do Sul, Brasil, 2005.

39. Borges, M. R.; Coutinho, E. L. M.; R. Bras. Ci. Solo 2004, 28, 543.

40. Schnitzer, M.; Soil Sci. Soc. Am. J. 1996, 33, 75.

41. Sodré, F. F.; Lenzi, E.; Costa, A. C. S.; Quim. Nova 2001, 24, 324.

42. Guilherme, L. R. G.; Anderson, S. J. Em Copper Sorption kinetics in two oxide-rich soils (Oxisols): effect of phosphate pretreatment; Jenne, E. A., ed.; Academic Presss: San Diego, 1998.

43. Ma, L. Q.; Rao, G. N.; J. Environ. Qual. 1997, 26, 259.

44. Basta, N. T.; Tabatabai, M. A.; Soil Sci. 1992, 156, 195.

45. Echeverria, J. C.; Morera, M. T.; Mazkiaran, C.; Environ. Pollut. 1998, $101,275$. 